\title{
Representation and Reasoning of Three-Dimensional Spatial Relationships Based on R5DOS-Intersection Model Representation and Reasoning Based on R5DOS Model
}

\author{
Jian Li, ${ }^{1,2}$ Weijian Zhang $\mathbb{D}^{1}{ }^{1}$ Yating $\mathrm{Hu} \mathbb{D}^{1,2}$ and Zhun Wang ${ }^{1}$ \\ ${ }^{1}$ College of Information Technology, Jilin Agricultural University, Changchun 130118, China \\ ${ }^{2}$ Bioinformatics Research Center of Jilin Province, Changchun 130118, China \\ Correspondence should be addressed to Yating Hu; huyating@jlau.edu.cn
}

Received 7 July 2020; Revised 7 September 2020; Accepted 13 September 2020; Published 7 October 2020

Academic Editor: Abd E. I.-Baset Hassanien

Copyright (C) 2020 Jian Li et al. This is an open access article distributed under the Creative Commons Attribution License, which permits unrestricted use, distribution, and reproduction in any medium, provided the original work is properly cited.

\begin{abstract}
This paper aims to disclose the compound topological and directional relationships of three simple regions in the three-dimensional (3D) space. For this purpose, the directional model and the 8-intersection model were coupled into an R5DOSintersection model and used to represent three simple regions in the 3D space. The matrices represented by the model were found to be complete and mutually exclusive. Then, a self-designed algorithm was adopted to solve the model, yielding 11,038 achievable topological and directional relationships. Compared with the minimum bounding rectangle (MBR) model, the proposed model boasts strong expressive power. Finally, our model was applied to derive the topological and directional relationships between simple regions $\mathrm{A}$ and $\mathrm{C}$ from the known relationships between simple regions $\mathrm{A}$ and $\mathrm{B}$ and those between $\mathrm{B}$ and $\mathrm{C}$. Based on the results, a compound relationship reasoning table was established for $\mathrm{A}$ and $\mathrm{C}$. The research results shed new light on the representation and reasoning of $3 \mathrm{D}$ spatial relationships.
\end{abstract}

\section{Introduction}

The reasoning of spatial relationship, a.k.a. spatial reasoning, can be implemented quantitatively or qualitatively. Qualitative spatial reasoning, aiming to represent and analyze spatial information, is an important tool in artificial intelligence $(\mathrm{AI})$, machine vision, robot navigation $[1,2]$, and geographic information system [3].

Over three decades, many theories and models have been developed for spatial reasoning. For instance, Randell et al. $[4,5]$ put forward the region connection calculus (RCC) theory. Egenhofer and Franzosa [6,7] proposed the theory of 4-intersection model and 9-intersection model. Li [8] derived a dynamic reasoning method for azimuth relationship.

In recent years, spatial reasoning has evolved rapidly, thanks to the emerging AI applications in image processing $[9,10]$, computer vision $[11,12]$, and model prediction [13]. However, most studies on spatial reasoning focus on the spatial relationships on two-dimensional (2D) planes rather than those in three-dimensional (3D) spaces. The 3D space contains too many information elements to be handled by ordinary reasoning methods.

At present, the relationships between objects in the 3D space are mostly solved by compound reasoning. The common approaches of compound reasoning include the compound reasoning of directional and topological relationships $[14,15]$ and the compound reasoning of directional and distance relationships [16]. Liu et al. [17] designed a 3D improved composite spatial relationship model (3DICSRM) in a large-scale environment and proposed a reasoning algorithm to solve that model. The accuracy of the 3D-ICSRM is very limited, and it considers the relationship between qualitative distance and direction. In 2016, Hou et al. [18] extended the convex tractable subalgebra into 3D space and used the BCD algorithm to calculate it. In 2019, Wang et al. [19] extended the oriented point relation algebra (OPRAm) model to 3D and proposed oriented point relation algebra in three-dimensional (OPRA3Dm) algorithm, which 
has certain practical significance. These two papers consider the direction relationship. In recent years, the literature mainly studies the relationship between the direction and qualitative distance, while there is less research on the direction and topological relationship. This article will focus on the direction and topological relationship to fill the gaps in this field.

This paper aims to disclose the compound topological and directional relationships of three simple regions in the $3 \mathrm{D}$ space. Firstly, the RCC-5 model was combined with a strong directional relationship model for two simple regions, based on the extended 4-intersection theory and spatial orientation relationship in RCC5. The combined model was used to identify the compound topological and azimuth relationships between two simple regions, and solved by a self-designed algorithm. Through programming, a total of 65 topological and directional relationships were obtained in the $3 \mathrm{D}$ space.

On this basis, the extended 4-intersection matrix was replaced with an 8 -intersection matrix to represent the $3 \mathrm{D}$ spatial topological and directional relationships between three simple regions. Then, it was found that the topological and directional relationships between the R5DOS-intersection model of two regions and three regions are complete and mutually exclusive. Further programming reveals a total of 11,038 topological and azimuth relationships between three simple regions in the $3 \mathrm{D}$ space and derives a simple

$$
\left(\begin{array}{cccc}
A^{o} \cap B^{o} \cap C^{o} & A^{o} \cap B^{o} \cap\left(C^{c}\right)^{o} & A^{o} \cap\left(B^{c}\right)^{o} \cap C^{o} & A^{o} \cap\left(B^{c}\right)^{o} \cap\left(C^{c}\right)^{o} \\
\left(A^{c}\right)^{o} \cap B^{o} \cap C^{o} & \left(A^{c}\right)^{o} \cap B^{o} \cap\left(C^{c}\right)^{o} & \left(A^{c}\right)^{o} \cap\left(B^{c}\right)^{o} \cap C^{o} & \left(A^{c}\right)^{o} \cap\left(B^{c}\right)^{o} \cap\left(C^{c}\right)^{o}
\end{array}\right) .
$$

The RCC theory fuels the research on spatial relationship models in the past three decades, giving birth to many new theories. Nonetheless, most of these theories target the 2D plane rather than the $3 \mathrm{D}$ space. Recently, there is a growing interest in the spatial relationship models of the 3D space, especially the compound reasoning of directional and topological relationships, and that of directional and distance relationships.

2.2. Orientation Model. Minimum bounding rectangle (MBR) is a commonly used model for directional relationship in space [18-20]. The MBR model, 8-direction model, and 16-direction model are shown in Figure 3 below. The MBR model is not consistent with human cognition of directions.

In 2010, He and Bian [21] came up with a special 8direction cone model (Figure 4), which divides the space into eight regions: NW, NE, EN, ES, SE, SW, WS, and $\mathrm{WN}$. Among them, NW and NE belong to the N direction, EN and ES belong to the E direction, SE and SW belong to the $\mathrm{S}$ direction, and $\mathrm{WS}$ and $\mathrm{WN}$ belong to the $\mathrm{W}$ direction.

The 8-direction cone model is easy to describe and recognize and is flexible in dealing with relationships in topological and directional relationship $R(A, C)$ from two sets of two simple regions $R(A, B)$ and $R(B, C)$.

\section{Materials and Methods}

2.1. RCC Theory. In 1992, Randell et al. [4, 5] proposed the RCC theory and established the RCC- 8 intersection model, which is a boundary-sensitive model. Based on the boundary-sensitive conditions, the RCC-5 intersection model can be derived (Figure 1).

In 1991 and 1995, Egenhofer et al. constructed an extended 4-intersection matrix, which covers two space objects $A$ and $B$, with $A^{\circ}$ being the interior of $A$ :

$$
\left(\begin{array}{cc}
A^{o} \cap B^{o} & A^{o} \cap\left(B^{c}\right)^{o} \\
\left(A^{o}\right)^{o} \cap B^{o} & \left(A^{o}\right)^{C} \cap\left(B^{c}\right)^{o}
\end{array}\right) .
$$

The value of each position set is either empty or nonempty. Then, the five kinds of relationships in the RCC-5 intersection model can be represented as the matrix in Table 1 and expressed as a set $R_{5}=\left\{\left(\begin{array}{llll}0 & 1 & 1 & 1\end{array}\right),\left(\begin{array}{llll}1 & 1 & 1 & 1\end{array}\right)\right.$, $\left.\left(\begin{array}{llll}1 & 0 & 1 & 1\end{array}\right),\left(\begin{array}{llll}1 & 0 & 0 & 1\end{array}\right),\left(\begin{array}{llll}1 & 1 & 0 & 1\end{array}\right)\right\}$.

For three simple areas $A, B$, and $C, R^{2}-\{\partial A \cup \partial B \cup \partial C\}$ can be partitioned into 8 parts (Figure 2).

The eight parts can be illustrated by an 8 -intersection matrix:

multiple dimensions. Compared with the 8-direction cone model, the16-direction cone model is also consistent with the human cognition of directions, but too complicated to express. Hence, the 8-direction cone model is more suitable for the reasoning of spatial relationships.

Considering its excellence in spatial segmentation, the 8direction cone model was coupled with the RCC-5 intersection model for compound reasoning of topological and azimuth relationships in the $3 \mathrm{D}$ space.

2.3. Model Construction. Any object in space is wrapped by an outer sphere $\odot A$ with a radius $r_{A}$ (Figure 5), that is, $\forall\left(x_{A}, y_{A}, z_{A}\right) \in \odot A$.

Taking the center of $\odot A$ as the origin of the rectangular coordinate system in space, the spatial Cartesian coordinate system can be established and the reference space can be divided into eight intervals by the $x$-, $y$-, and $z$-axes. Each interval is called a hexagram limit Oct $=\{1,2,3,4,5,6,7,8\}$ (Figure 6).

Suppose $n$ points $B_{i}\left(x_{i}, y_{i}, z_{i}\right) \in(i=1,2, \ldots, n)$ are scattered in the space. The centroid $b\left(x_{b}, y_{b}, z_{b}\right)$ of the point set $B=\left\{B_{1}, B_{2}, \ldots, B_{n}\right\}$ can be obtained by $k$-means clustering (KMC) [20] and treated as the center of the sphere of point set $B$ : 


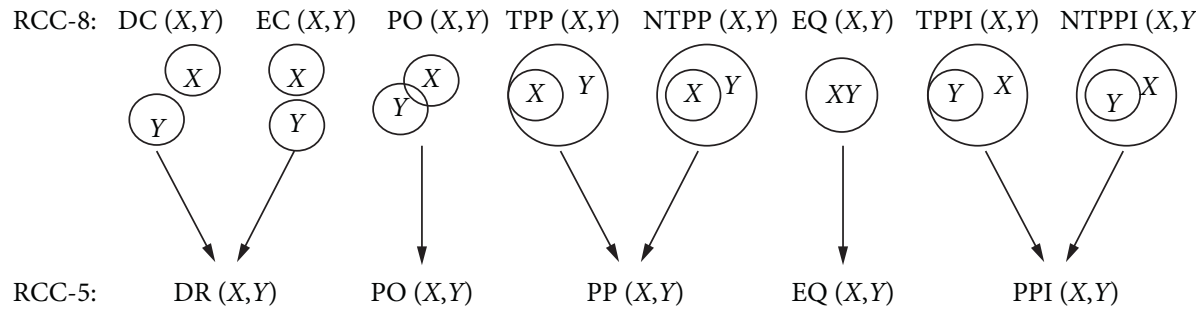

Figure 1: The relationships between RCC-8 and RCC-5 intersection models.

TABLE 1: Matrix representation of the RCC-5 relationships.

\begin{tabular}{|c|c|c|c|c|c|}
\hline RCC5 relationships & $\mathrm{DR}(A, B)$ & $\mathrm{PO}(A, B)$ & $\mathrm{PP}(A, B)$ & EQ $(A, B)$ & PPI $(A, B)$ \\
\hline Extended 4-intersection matrix representation & $\left(\begin{array}{ll}0 & 1 \\
1 & 1\end{array}\right)$ & $\left(\begin{array}{ll}1 & 1 \\
1 & 1\end{array}\right)$ & $\left(\begin{array}{ll}1 & 0 \\
1 & 1\end{array}\right)$ & $\left(\begin{array}{ll}1 & 0 \\
0 & 1\end{array}\right)$ & $\left(\begin{array}{ll}1 & 1 \\
0 & 1\end{array}\right)$ \\
\hline
\end{tabular}

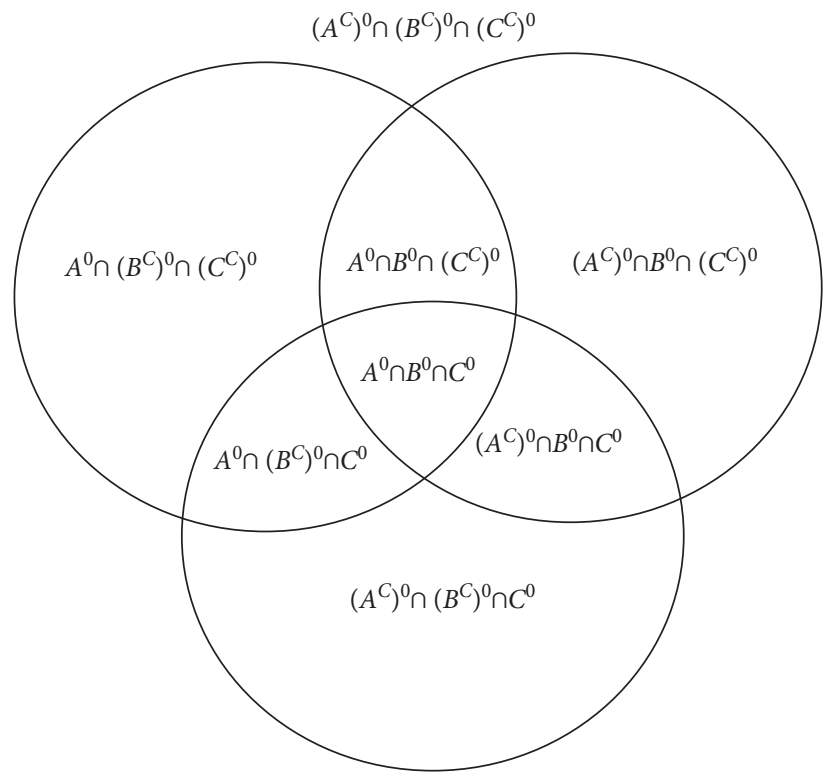

FIgURE 2: The spatial partition of three simple areas.

\begin{tabular}{c|c|c} 
NW & N & NE \\
\hline W & O & E \\
\hline SW & S & SE
\end{tabular}

(a)

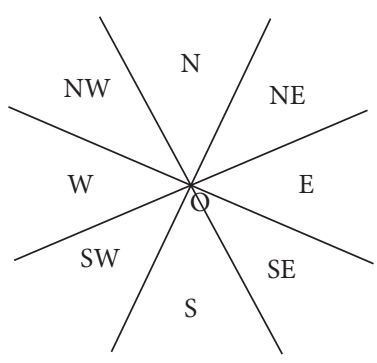

(b)

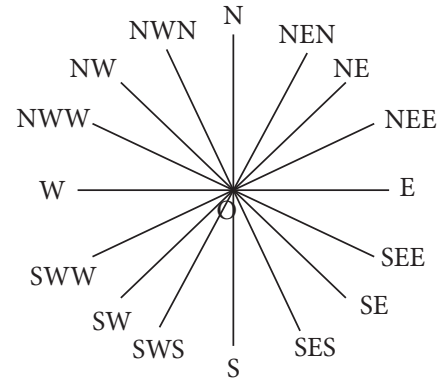

(c)

FIgUre 3: (a) The MBR model, (b) 8-direction model, and (c) 16-direction model. 


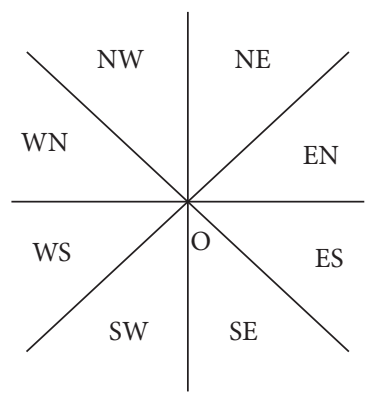

FIGURE 4: The 8-direction cone model.

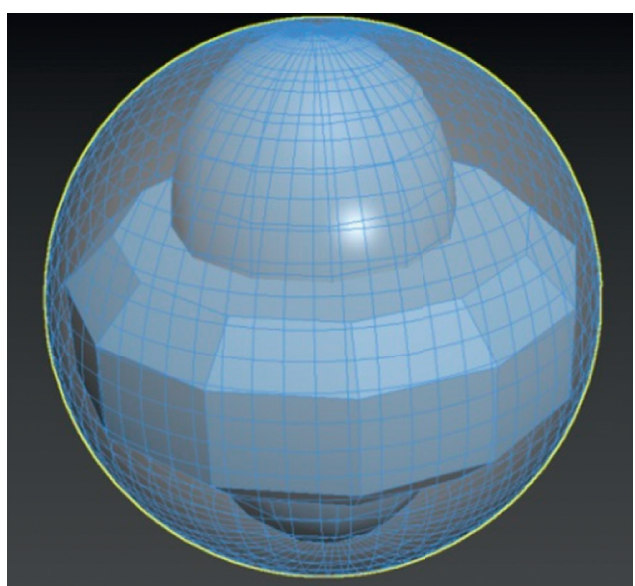

FIgURE 5: The outer sphere.

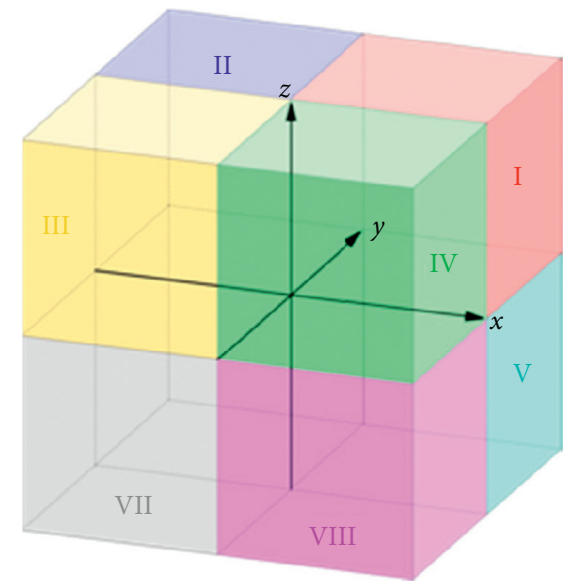

Figure 6: The hexagram limits.

$$
\left\{\begin{array}{l}
x_{b}=\frac{x_{q B}+x_{p B}}{2}, \\
y_{b}=\frac{y_{q B}+y_{p B}}{2}, \\
z_{b}=\frac{z_{q B}+z_{p B}}{2} .
\end{array}\right.
$$

The outer sphere $B$ completely covers the $n$ points: $\forall\left(x_{B i}, y_{B i}, z_{B i}\right) \in \odot B$. Similarly, the outer sphere $C$ for point set $C$ can be defined as follows:

$$
\left\{\begin{array}{l}
x_{C}=\frac{x_{q C}+x_{p C}}{2}, \\
y_{C}=\frac{y_{q C}+y_{p C}}{2}, \quad \forall\left(x_{C i}, y_{C i}, z_{C i}\right) \in \odot C, \\
z_{C}=\frac{z_{q C}+z_{p C}}{2} .
\end{array}\right.
$$

If it is impossible to find the outer sphere of the space object, the object can be treated as an irregular convex object. Then, five planes $\pi_{1}: y=0, \pi_{2}: x=0, \pi_{3}: z=0$, $\pi_{4}: y=z$, and $\pi_{5}: y=-z$, can be inserted into the rectangular coordinate system in space (Figure 7).

Then, the $3 \mathrm{D}$ space can be represented as Dic $=\{N E, E N, E S, S E, S W, W S, W N, N W\}$. The angle corresponding to each region can be described as follows:

$$
\left\{\begin{array}{l}
\theta_{\mathrm{NE}} \in\left[0, \frac{\pi}{4}\right), \\
\theta_{\mathrm{EN}} \in\left[\frac{\pi}{4}, \frac{\pi}{2}\right), \\
\theta_{\mathrm{ES}} \in\left[\frac{\pi}{2}, \frac{3 \pi}{4}\right), \\
\theta_{\mathrm{SE}} \in\left[\frac{3 \pi}{4}, \pi\right), \\
\theta_{\mathrm{SW}} \in\left[\pi, \frac{5 \pi}{4}\right), \\
\theta_{\mathrm{WS}} \in\left[\frac{5 \pi}{4}, \frac{3 \pi}{2}\right), \\
\theta_{\mathrm{WN}} \in\left[\frac{3 \pi}{2}, \frac{7 \pi}{4}\right), \\
\theta_{\mathrm{NW}} \in\left[\frac{7 \pi}{4}, 2 \pi\right],
\end{array}\right.
$$

where $\theta$ is the dihedral angle of the plane $\pi_{i}(i=1,2,3,4,5)$. Adding the set of hexagram limits Oct $=\{1,2,3,4,5,6,7,8\}$, the space can be divided into 16 regions:

$$
\mathrm{DO}=\left(\begin{array}{cccc}
1 \mathrm{NE} & 2 \mathrm{NE} & 3 \mathrm{NW} & 4 \mathrm{NW} \\
1 \mathrm{EN} & 2 \mathrm{EN} & 3 \mathrm{NW} & 4 \mathrm{NW} \\
5 \mathrm{ES} & 6 \mathrm{ES} & 7 \mathrm{WS} & 8 \mathrm{WS} \\
5 \mathrm{SE} & 6 \mathrm{SE} & 7 \mathrm{SW} & 8 \mathrm{SW}
\end{array}\right),
$$

where $\mathrm{DO}$ is the set of $3 \mathrm{D}$ regions and their hexagram limits. If the center of outer sphere $B$ exists in region $1 \mathrm{NE}$, then $B$ strongly exists in that region, denoted as s1NE. If outer sphere B partly exists in region $2 \mathrm{NE}$, then $B$ weakly exists in that region, denoted as w $2 \mathrm{NE}$. We let " 0 " indicate that there is no object in the area, " 1 " indicates that the object "strongly 


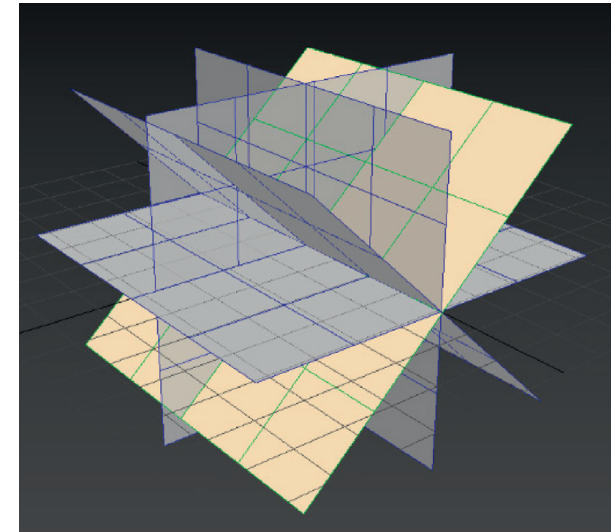

Figure 7: The insertion of five planes.
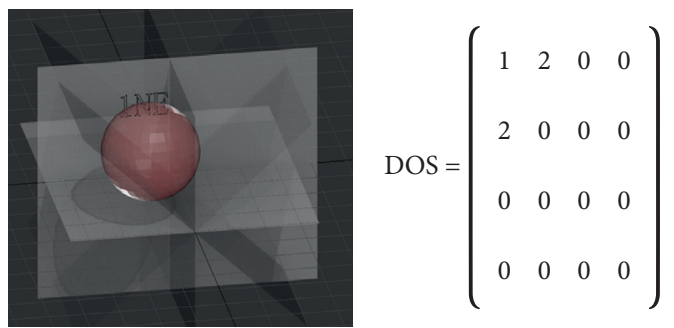

Figure 8: Examples of "weakly presence".

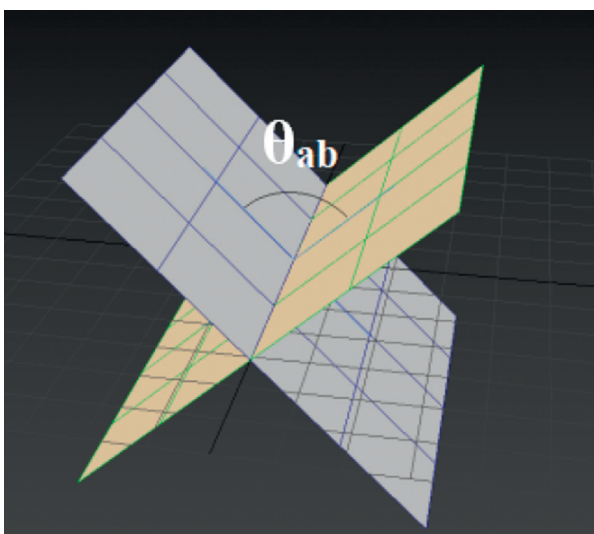

Figure 9: The dihedral angle.

exists" in this area, and " 2 " indicates that the object "weakly exists" in this area. An example is shown in Figure 8:

$$
\operatorname{DOS}=\left(\begin{array}{cccc}
1 & 2 & 0 & 0 \\
2 & 0 & 0 & 0 \\
0 & 0 & 0 & 0 \\
0 & 0 & 0 & 0
\end{array}\right) \text {. }
$$

For simplicity, only strong existence scenarios were considered. Then, the set of regions, where $B$ strongly exists, can be defined as follows:

$$
\mathrm{DOS}=\left(\begin{array}{cccc}
s 1 \mathrm{NE} & s 2 \mathrm{NE} & s 3 \mathrm{NW} & s 4 \mathrm{NW} \\
s 1 \mathrm{EN} & s 2 \mathrm{EN} & s 3 \mathrm{NW} & s 4 \mathrm{NW} \\
s 5 \mathrm{ES} & s 6 \mathrm{ES} & s 7 \mathrm{WS} & s 8 \mathrm{WS} \\
s 5 \mathrm{SE} & s 6 \mathrm{SE} & s 7 \mathrm{SW} & s 8 \mathrm{SW}
\end{array}\right),
$$

where

$$
\begin{aligned}
& \begin{cases}s 1 \mathrm{NE} ; x_{b} \geq 0, y_{b} \geq 0, z_{b} \geq 0, & \theta_{o b} \in\left[0, \frac{\pi}{4}\right), \\
s 2 \mathrm{NE} ; x_{b}<0, y_{b} \geq 0, z_{b} \geq 0, & \theta_{o b} \in\left[0, \frac{\pi}{4}\right), \\
s 1 \mathrm{EN} ; x_{b} \geq 0, y_{b} \geq 0, z_{b} \geq 0, & \theta_{o b} \in\left[\frac{\pi}{4}, \frac{\pi}{2}\right), \\
s 2 \mathrm{EN} ; x_{b}<0, y_{b} \geq 0, z_{b} \geq 0, & \theta_{o b} \in\left[\frac{\pi}{4}, \frac{\pi}{2}\right),\end{cases}
\end{aligned}
$$

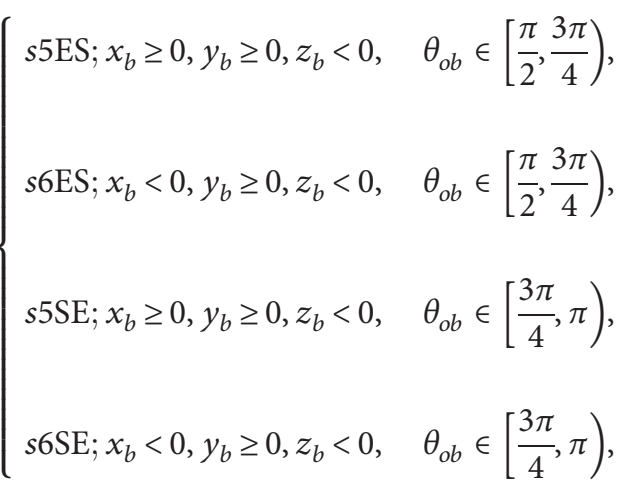

$$
\begin{aligned}
& s 8 S W ; x_{b} \geq 0, y_{b} \geq 0, z_{b}<0, \quad \theta_{o b} \in\left[\pi, \frac{5 \pi}{4}\right), \\
& s 7 S W ; x_{b} \geq 0, y_{b} \geq 0, z_{b} \geq 0, \quad \theta_{o b} \in\left[\pi, \frac{5 \pi}{4}\right), \\
& s 8 \mathrm{WS} ; x_{b} \geq 0, y_{b}<0, z_{b}<0, \quad \theta_{o b} \in\left[\frac{5 \pi}{4}, \frac{3 \pi}{2}\right) \text {, } \\
& s 7 \mathrm{WS} ; x_{b}<0, y_{b}<0, z_{b}<0, \quad \theta_{o b} \in\left[\frac{5 \pi}{4}, \frac{3 \pi}{2}\right), \\
& s 4 \mathrm{WN} ; x_{b} \geq 0, y_{b}<0, z_{b} \geq 0, \quad \theta_{o b} \in\left[\frac{3 \pi}{2}, \frac{7 \pi}{4}\right), \\
& s 3 \mathrm{WN} ; x_{b}<0, y_{b}<0, z_{b} \geq 0, \quad \theta_{o b} \in\left[\frac{3 \pi}{2}, \frac{7 \pi}{4}\right), \\
& s 4 \mathrm{NW} ; x_{b} \geq 0, y_{b}<0, z_{b} \geq 0, \quad \theta_{o b} \in\left[\frac{7 \pi}{4}, 2 \pi\right), \\
& s 3 \mathrm{NW} ; x_{b}<0, y_{b}<0, z_{b} \geq 0, \quad \theta_{o b} \in\left[\frac{7 \pi}{4}, 2 \pi\right)
\end{aligned}
$$


where $\theta_{o b}$ the dihedral angle formed by planes $\pi_{o b}$ and $\pi_{1}$, which is perpendicular to the $x$-axis and passes the straight line $a b$ (Figure 9).

For two regions, the extended 4-intersection matrix can be introduced to the DOS:

$$
R 5_{2} \mathrm{DOS}=\left(\begin{array}{cccc}
A^{o} \cap B^{o} & A^{o} \cap\left(B^{c}\right)^{o} & \left(A^{c}\right)^{o} \cap B^{o} & \left(A^{c}\right)^{o} \cap\left(B^{c}\right)^{o} \\
s 1 \mathrm{NE} & s 2 \mathrm{EN} & s 3 \mathrm{NW} & s 4 \mathrm{NW} \\
s 1 \mathrm{EN} & s 2 \mathrm{EN} & s 3 \mathrm{NW} & s 4 \mathrm{NW} \\
s 5 \mathrm{ES} & s 6 \mathrm{ES} & s 7 \mathrm{WS} & s 8 \mathrm{WS} \\
s 5 \mathrm{SE} & s 6 \mathrm{SE} & s 7 \mathrm{SW} & s 8 \mathrm{SW}
\end{array}\right) .
$$

For three regions, the 8-intersection matrix can be introduced to the DOS:

$$
R 5_{3} \mathrm{DOS}=\left(\begin{array}{cccc}
A^{o} \cap B^{o} \cap C^{o} & A^{o} \cap B^{o} \cap\left(C^{c}\right)^{o} & A^{o} \cap\left(B^{c}\right)^{o} \cap C^{o} & A^{o} \cap\left(B^{c}\right)^{o} \cap\left(C^{c}\right)^{o} \\
\left(A^{c}\right)^{o} \cap B^{o} \cap C^{o} & \left(A^{c}\right)^{o} \cap B^{o} \cap\left(C^{c}\right)^{o} & \left(A^{c}\right)^{o} \cap\left(B^{c}\right)^{o} \cap C^{o} & \left(A^{c}\right)^{o} \cap\left(B^{c}\right)^{o} \cap\left(C^{c}\right)^{o} \\
s 1 \mathrm{NE} & s 2 \mathrm{NE} & s 3 \mathrm{NW} & s 4 \mathrm{NW} \\
s 1 \mathrm{EN} & s 2 \mathrm{EN} & s 3 \mathrm{NW} & s 4 \mathrm{NW} \\
s 5 \mathrm{ES} & s 6 \mathrm{ES} & s 7 \mathrm{WS} & s 8 \mathrm{WS} \\
s 5 \mathrm{SE} & s 6 \mathrm{SE} & s 7 \mathrm{SW} & s 8 \mathrm{SW}
\end{array}\right) .
$$

Our model consists of two layers: the first layer is the topological relationship $R_{5}$ layer, and the second layer is the orientation relationship DOS layer. Then, the following $\varepsilon(\mathrm{DOS})= \begin{cases}1, & \text { the enter of the outer sphere } B \text { exists in this region, } \\ 0, & \text { otherwise. }\end{cases}$ definition can be derived.

Definition 1. For the orientation relationship layer, there is

Suppose

$$
\varepsilon\left(R 5_{2} \mathrm{DOS}\right)=\left(\begin{array}{cccc}
\varepsilon\left(A^{o} \cap B^{o}\right) & \varepsilon\left(A^{o} \cap\left(B^{c}\right)^{o}\right) & \varepsilon\left(\left(A^{c}\right)^{o} \cap B^{o}\right) & \varepsilon\left(\left(A^{c}\right)^{o} \cap\left(B^{c}\right)^{o}\right) \\
\varepsilon(s 1 N E) & \varepsilon(s 2 E N) & \varepsilon(s 3 N W) & \varepsilon(s 4 N W) \\
\varepsilon(s 1 E N) & \varepsilon(s 2 E N) & \varepsilon(s 3 N W) & \varepsilon(s 4 N W) \\
\varepsilon(s 5 E S) & \varepsilon(s 6 E S) & \varepsilon(s 7 W S) & \varepsilon(s 8 W S) \\
\varepsilon(s 5 S E) & \varepsilon(s 6 S E) & \varepsilon(s 7 S W) & \varepsilon(s 8 S W)
\end{array}\right) .
$$

For any two simple regions $A$ and $B$, it is possible to obtain a $5 \times 4^{c} 0-1$ matrix. In theory, a total of $2^{20}$ matrices could be acquired, which correspond to $2^{20}$ topological and directional relationships in the 3D space:

$$
R 5_{3} \mathrm{DOS}=\left(\begin{array}{cccc}
\varepsilon\left(A^{o} \cap B^{o} \cap C^{o}\right) & \varepsilon\left(A^{o} \cap B^{o} \cap\left(C^{c}\right)^{o}\right) & \varepsilon\left(A^{o} \cap\left(B^{c}\right)^{o} \cap C^{o}\right) & \varepsilon\left(A^{o} \cap\left(B^{c}\right)^{o} \cap\left(C^{c}\right)^{o}\right) \\
\varepsilon\left(\left(A^{c}\right)^{o} \cap B^{o} \cap C^{o}\right) & \varepsilon\left(\left(A^{c}\right)^{o} \cap B^{o} \cap\left(C^{c}\right)^{o}\right) & \varepsilon\left(\left(A^{c}\right)^{o} \cap\left(B^{c}\right)^{o} \cap C^{o}\right) & \varepsilon\left(\left(A^{c}\right)^{o} \cap\left(B^{c}\right)^{o} \cap\left(C^{c}\right)^{o}\right) \\
\varepsilon(s 1 \mathrm{NE}) & \varepsilon(s 2 \mathrm{NE}) & \varepsilon(s 3 \mathrm{NW}) & \varepsilon(s 4 \mathrm{NW}) \\
\varepsilon(s 1 \mathrm{EN}) & \varepsilon(s 2 \mathrm{EN}) & \varepsilon(s 3 \mathrm{NW}) & \varepsilon(s 4 \mathrm{NW}) \\
\varepsilon(s 5 \mathrm{ES}) & \varepsilon(s 6 \mathrm{ES}) & \varepsilon(s 7 \mathrm{WS}) & \varepsilon(s 8 \mathrm{WS}) \\
\varepsilon(s 5 \mathrm{SE}) & \varepsilon(s 6 \mathrm{SE}) & \varepsilon(s 7 \mathrm{SW}) & \varepsilon(s 8 \mathrm{SW})
\end{array}\right) .
$$


Based on the topological relationship between outer spheres $B$ and $C$, the existence of the centers of the two spheres can be described in two cases.
Case 1. Only the center of one outer sphere exists in the current region:

$$
\varepsilon(\mathrm{DOS})= \begin{cases}0, & \text { no center exists in the current region, } \\ 1, & \text { the center of outer sphere } B \text { exists in the current region, } \\ 2, & \text { the center of outer sphere } C \text { exists in the current region. }\end{cases}
$$

Case 2. The centers of both outer spheres exist in the current region:

$$
\varepsilon(\mathrm{DOS})= \begin{cases}0, & \text { no center exists in the current region, } \\ 1, & \text { the centers of both outer spheres exist in the current region. }\end{cases}
$$

According to the above conditions, $2^{8} \times 3^{16}$ matrices could be obtained theoretically, which correspond to $2^{8} \times 3^{16}$ topological and directional relationships in the $3 \mathrm{D}$ space.

\subsection{Model Properties}

Definition 2. In layer $R_{5}$, any $m \times n$-order 0-1 matrices $A=$ $\left(a_{i j}\right)_{m \times n}$ and $B=\left(b_{i j}\right)_{m \times n}$ can be defined as $A \cup B=$ $\left(a_{i j} \vee b_{i j}\right)_{m \times n}$. Then, a 0-1 diagonal matrix can be established as Table 2.

The following proposition can be derived from Table 2:

Proposition 1. $\varepsilon(A \cup B)=\varepsilon(A) \vee \mathcal{E}(B)$.

For $\quad R_{5}=\left\{\begin{array}{llll}0 & 1 & 1 & 1\end{array}\right),\left(\begin{array}{llll}1 & 1 & 1 & 1\end{array}\right),\left(\begin{array}{llll}1 & 0 & 1 & 1\end{array}\right)$, $\left.\left(\begin{array}{llll}1 & 0 & 0 & 1\end{array}\right),\left(\begin{array}{llll}1 & 1 & 0 & 1\end{array}\right)\right\}, R(A, B)$ is the element that corresponds to the topological relationship $R_{5}$ between any two simple regions $A$ and $B$.

Then, the following theorem can be obtained.

Theorem 1. For simple regions $A, B$, and $C$, there exists $\left(\begin{array}{cc}\varepsilon\left(A^{o} \cap B^{o}\right) & \varepsilon\left(A^{o} \cap\left(B^{c}\right)^{o}\right) \\ \varepsilon\left(\left(A^{c}\right)^{o} \cap B^{o}\right) & \varepsilon\left(\left(A^{o}\right)^{C} \cap\left(B^{c}\right)^{o}\right)\end{array}\right)=R(A, B) \in R_{5}$. Similarly, there exists $\left(\begin{array}{cc}\varepsilon\left(A^{o} \cap C^{o}\right) & \varepsilon\left(A^{o} \cap\left(C^{c}\right)^{o}\right) \\ \varepsilon\left(\left(A^{c}\right)^{o} \cap C^{o}\right) & \varepsilon\left(\left(A^{o}\right)^{C} \cap\left(C^{c}\right)^{o}\right)\end{array}\right)=R(A, C) \in R_{5}$.

Theorem 2. In the 3D space given by the R5DOS-intersection matrix, the topological relationship between the three simple regions is mutually exclusive and complete. The DOS space of the R5DOS-intersection matrix, which consists of 16 regions, is a half-open, half-closed interval with mutual exclusion. That is, for any three simple regions $A, B$, and $C$ in the $3 D$ space, there exists only one relationship satisfied by the ordered pair $\langle A, B, C\rangle$.

Proof. For any three simple regions $A, B$, and $C$, the 8 and 16 regions divided by the 8 -interesection matrix are disjoint. The
0-1 matrix of three simple regions uniquely corresponds to the matrix derived from the R5DOS-intersection model. In other words, the three regions have the relationship represented by this matrix so that the R5DOS-intersection matrix model gives a complete topological relationship in the 3D space.

Then, it is assumed that the topological relationship between $A, B$, and $C$ corresponds to two matrices $R 5_{3} \mathrm{DOSa}$ and $R 5_{3} \mathrm{DOSb}$ and can be induced by the R $5_{3} \mathrm{DOS}$-intersection model. Then, there exists $1 \leq i \leq 24$ such that $R 5_{3} \mathrm{DO}_{S} a_{i} \neq R 5_{3} \mathrm{DO}_{S} b_{i} . \quad$ If $\quad i=1, R 5_{3} \mathrm{DOSa}_{i}=0$ and $R 5$ ${ }_{3} \mathrm{DOSb}_{i}=1,{ }^{A^{\circ}} \cap B^{B^{\circ}} \cap C^{\circ}$ is both empty and nonempty, which is obviously contradictory. Hence, the above theorem was proved valid.

2.5. Constraints on Two Simple Regions. Theoretically, two simple regions might correspond to $2^{20}$ matrices, but there must be $0-1$ matrices that cannot be realized. Therefore, the following constraints were designed on two simple regions.

Constraint 1: to correspond to a real-world topological relationship, the 0-1 matrix of layer $R_{5}$ must belong to one of the five cases: $R_{5}=\left\{\begin{array}{llll}0 & 1 & 1 & 1\end{array}\right)$,

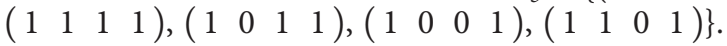

Constraint 2: if layer $R_{5}$ satisfies $R 5=\left(\begin{array}{llll}1 & 0 & 0 & 1\end{array}\right)$, that is, outer spheres $A$ and $B$ are equal, then the 0-1 matrix of the DOS layer is DOS $=\left(\begin{array}{llll}0 & 0 & 0 & 0 \\ 0 & 0 & 0 & 0 \\ 0 & 0 & 0 & 0 \\ 0 & 0 & 0 & 0\end{array}\right)$. This means, when outer spheres $A$ and $B$ are equal, the center $b$ of outer sphere $B$ is $G$, which coincides with that of outer sphere $A: b\left(x_{b}, y_{b}, z_{b}\right)=(0,0,0)$.

Constraint 3: since the 16 regions are disjoint, they must be mutually exclusive and complete. If $R(A, B)$ does not fall at the center of outer sphere $B$, it can only exist in one of these regions. In the DOS layer, an outer sphere only exists in one of the 16 intervals. In this way, 
TABLE 2: The 0-1 diagonal matrix.

\begin{tabular}{lll}
\hline$\vee$ & 0 & 1 \\
\hline 0 & 0 & 1 \\
1 & 1 & 1 \\
\hline
\end{tabular}

a total of 65 directional and topological relationships can be obtained (Table 3 ).

Case 1

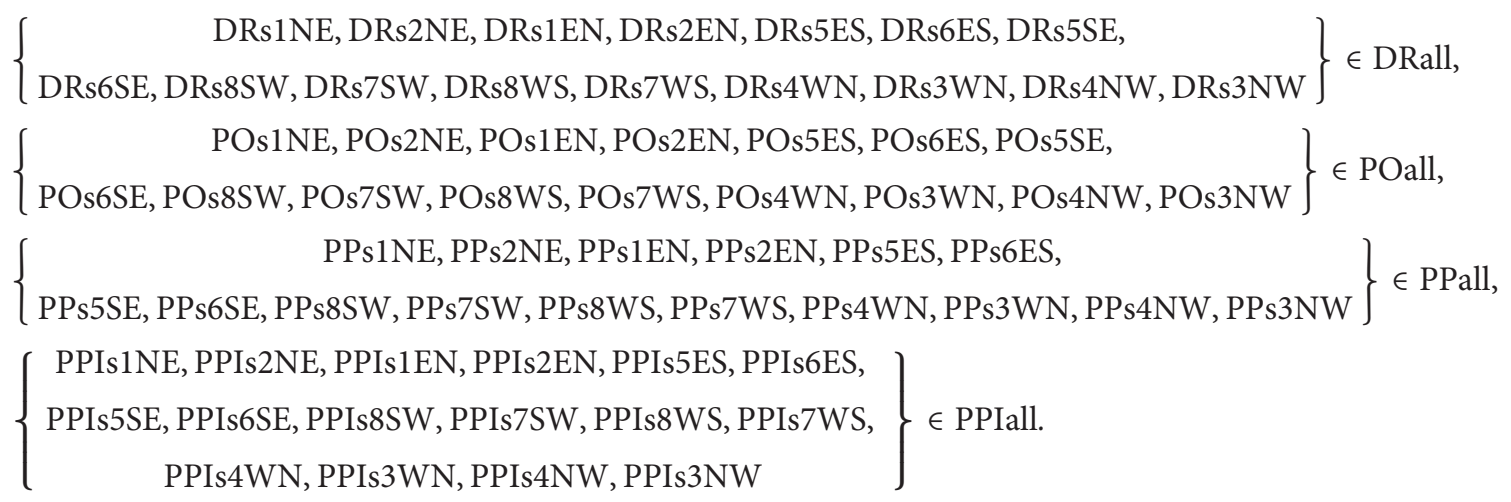

Case 2

$\{$ DRall, POall, PPall, EQ, PPIall $\} \in \Phi$.

Constraint 1: to uniquely correspond to the topological and directional relationships in the $3 \mathrm{D}$ space, a $R 5_{3} \mathrm{DOS}$ matrix must satisfy the following conditions.

2.6. Constraints on Three Simple Regions. The following constraints were designed on three simple regions.

Definition 3

$$
\begin{aligned}
& \left(\begin{array}{cc}
\varepsilon\left(A^{o} \cap B^{o} \cap C^{o}\right) & \varepsilon\left(A^{o} \cap\left(B^{c}\right)^{o} \cap C^{o}\right) \\
\varepsilon\left(\left(A^{c}\right)^{o} \cap B^{o} \cap C^{o}\right) & \varepsilon\left(\left(A^{c}\right)^{o} \cap\left(B^{c}\right)^{o} \cap C^{o}\right)
\end{array}\right) \vee\left(\begin{array}{cc}
\varepsilon\left(A^{o} \cap B^{o} \cap\left(C^{c}\right)^{o}\right) & \varepsilon\left(A^{o} \cap\left(B^{c}\right)^{o} \cap\left(C^{c}\right)^{o}\right) \\
\left(\varepsilon\left(A^{c}\right)^{o} \cap B^{o} \cap\left(C^{c}\right)^{o}\right) & \left(\varepsilon\left(A^{C}\right)^{o} \cap\left(B^{c}\right)^{o} \cap\left(C^{c}\right)^{o}\right)
\end{array}\right) \\
& R(A, B)=\left(\begin{array}{cc}
\varepsilon\left(A^{o} \cap B^{o}\right) & \varepsilon\left(A^{o} \cap\left(B^{c}\right)^{o}\right) \\
\left(\varepsilon\left(A^{c}\right)^{o} \cap B^{o}\right) & \left(\varepsilon\left(A^{o}\right)^{C} \cap\left(B^{c}\right)^{o}\right)
\end{array}\right) \in R_{5} \\
& \left(\begin{array}{cc}
\varepsilon\left(A^{o} \cap B^{o} \cap C^{o}\right) & \varepsilon\left(A^{o} \cap B^{o} \cap\left(C^{c}\right)^{o}\right) \\
\varepsilon\left(\left(A^{c}\right)^{o} \cap B^{o} \cap C^{o}\right) & \varepsilon\left(\left(A^{c}\right)^{o} \cap B^{o} \cap\left(C^{c}\right)^{o}\right)
\end{array}\right) \vee\left(\begin{array}{cc}
\varepsilon\left(A^{o} \cap\left(B^{c}\right)^{o} \cap C^{o}\right) & \varepsilon\left(A^{o} \cap\left(C^{c}\right)^{o} \cap\left(C^{c}\right)^{o}\right) \\
\varepsilon\left(\left(A^{c}\right)^{o} \cap\left(B^{c}\right)^{o} \cap C^{o}\right) & \varepsilon\left(\left(A^{c}\right)^{o} \cap\left(B^{c}\right)^{o} \cap\left(C^{c}\right)^{o}\right)
\end{array}\right), \\
& R(A, C)=\left(\begin{array}{cc}
\varepsilon\left(A^{o} \cap C^{o}\right) & \varepsilon\left(A^{o} \cap\left(C^{c}\right)^{o}\right) \\
\varepsilon\left(\left(A^{c}\right)^{o} \cap C^{o}\right) & \varepsilon\left(\left(A^{o}\right)^{C} \cap\left(C^{c}\right)^{o}\right)
\end{array}\right) \in R_{5}, \\
& \begin{array}{l}
\quad\left(\begin{array}{cc}
\varepsilon\left(A^{o} \cap B^{o} \cap C^{o}\right) & \varepsilon\left(A^{o} \cap B^{o} \cap\left(C^{c}\right)^{o}\right) \\
\varepsilon\left(A^{o} \cap\left(B^{c}\right)^{o} \cap C^{o}\right) & \varepsilon\left(A^{o} \cap\left(B^{c}\right)^{o} \cap\left(C^{c}\right)^{o}\right)
\end{array}\right) \vee\left(\begin{array}{cc}
\varepsilon\left(\left(A^{c}\right)^{o} \cap B^{o} \cap C^{o}\right) & \varepsilon\left(\left(A^{c}\right)^{o} \cap B^{o} \cap\left(C^{c}\right)^{o}\right) \\
\varepsilon\left(\left(A^{c}\right)^{o} \cap\left(B^{c}\right)^{o} \cap C^{o}\right) & \varepsilon\left(\left(A^{C}\right)^{o} \cap\left(B^{c}\right)^{o} \cap\left(C^{c}\right)^{o}\right)
\end{array}\right) \\
R(B, C)=\left(\begin{array}{cc}
\varepsilon\left(B^{o} \cap C^{o}\right) & \varepsilon\left(B^{o} \cap\left(C^{c}\right)^{o}\right) \\
\varepsilon\left(\left(B^{c}\right)^{o} \cap C^{o}\right) & \varepsilon\left(\left(B^{o}\right)^{C} \cap\left(C^{c}\right)^{o}\right)
\end{array}\right) \in R_{5} .
\end{array} \\
& \begin{array}{l}
\left(\begin{array}{cc}
\varepsilon\left(A^{o} \cap B^{o} \cap C^{o}\right) & \varepsilon\left(A^{o} \cap B^{o} \cap\left(C^{c}\right)^{o}\right) \\
\varepsilon\left(A^{o} \cap\left(B^{c}\right)^{o} \cap C^{o}\right) & \varepsilon\left(A^{o} \cap\left(B^{c}\right)^{o} \cap\left(C^{c}\right)^{o}\right)
\end{array}\right) \vee\left(\begin{array}{cc}
\varepsilon\left(\left(A^{c}\right)^{o} \cap B^{o} \cap C^{o}\right) & \varepsilon\left(\left(A^{c}\right)^{o} \cap B^{o} \cap\left(C^{c}\right)^{o}\right) \\
\varepsilon\left(\left(A^{c}\right)^{o} \cap\left(B^{c}\right)^{o} \cap C^{o}\right) & \varepsilon\left(\left(A^{C}\right)^{o} \cap\left(B^{c}\right)^{o} \cap\left(C^{c}\right)^{o}\right)
\end{array}\right) \\
R(B, C)=\left(\begin{array}{cc}
\varepsilon\left(B^{o} \cap C^{o}\right) & \varepsilon\left(B^{o} \cap\left(C^{c}\right)^{o}\right) \\
\varepsilon\left(\left(B^{c}\right)^{o} \cap C^{o}\right) & \varepsilon\left(\left(B^{o}\right)^{C} \cap\left(C^{c}\right)^{o}\right)
\end{array}\right) \in R_{5} .
\end{array}
\end{aligned}
$$




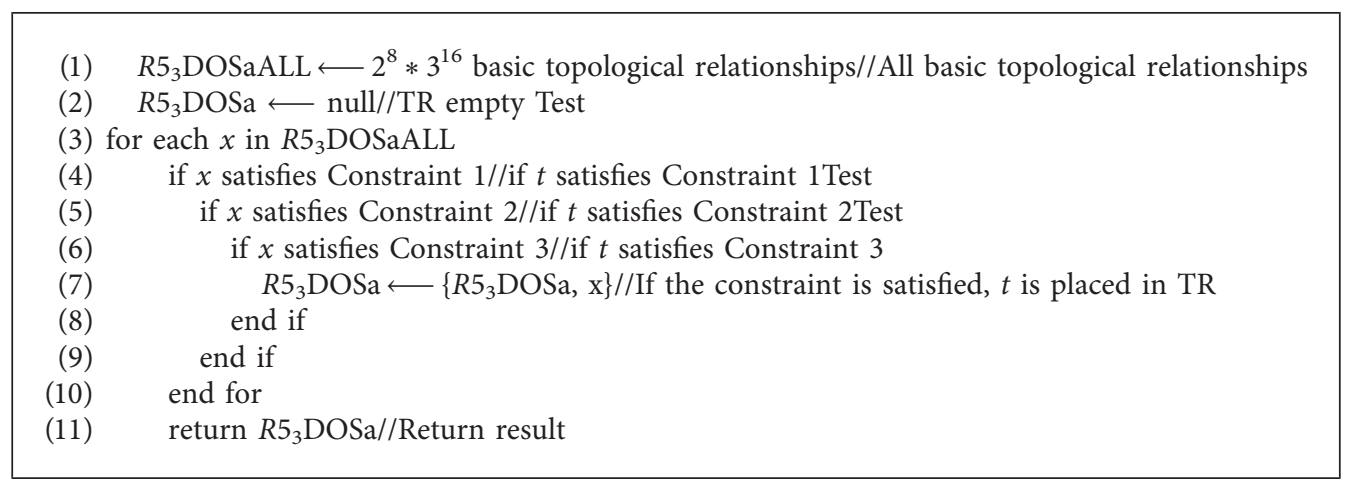

Algorithm 1

Constraint 2: since all three simple regions are bounded, $\left(A^{C}\right)^{\circ} \cap\left(B^{C}\right)^{\circ} \cap\left(C^{C}\right)^{\circ}$ is always 1 .

From Constraints 1 and 2, it can be inferred that layer $R_{5}$ has 109 topological relationships for any three simple regions in the $3 \mathrm{D}$ space.

Constraint 3: after adding the orientation relationship, some topological relationships are not satisfied in the orientation regions. In some topological relationships, the center of an outer sphere will change with that of the other outer spheres. For instance, if layer $R_{5}=\left(\begin{array}{llll}0 & 1 & 0 & 1 \\ 1 & 0 & 0 & 1\end{array}\right)$, the outer sphere $B$ will change with the overlap between outer spheres $A$ and $C$ (Figure 10). Case 1: if the $A, B$, and $C$ are equal, they can be regarded as one area:

$$
\text { DOS }=\left(\begin{array}{llll}
0 & 0 & 0 & 0 \\
0 & 0 & 0 & 0 \\
0 & 0 & 0 & 0 \\
0 & 0 & 0 & 0
\end{array}\right) .
$$

Case 2: if any two of the three simple regions are equal, the ternary region can be regarded as a binary region with only one 1 in the DOS layer.

Case 3: if any two of the three simple regions are inclusive or noninclusive, the ternary region can be regarded as a binary region when any two regions intersect and the sum of layer $R_{5}$ is 4 .

Case 4: if only one of the three simple regions is inclusive or noninclusive, the ternary region can be regarded as a binary region when any two regions intersect and the sum of layer $R_{5}$ is 5 .

Case 5: if the three simple regions are disjoint, the ternary region can be regarded as a binary region when any two regions intersect and the sum of layer $R_{5}$ is 5 .

Case 6: if simple regions $B$ and $C$ are inclusive or noninclusive and separated from $A$, then the center of the $A$ can only fall within $B$ and $C$ :

$$
\left(x_{\text {in }}, y_{\text {in }}, z_{\text {in }}\right) \in P_{\text {out }} .
$$

For a ternary reference object in the $3 \mathrm{D}$ space, there are theoretically $2^{8} \times 3^{16}$ matrices. Under the above constraints, a total of 11,038 matrices were obtained after removing the nonexistent scenarios.

2.7. Topological Relationship Algorithm for 3 Simple Regions in the 3D Space. The topological relationship algorithm for 3 simple regions in the $3 \mathrm{D}$ space can be implemented in the following steps.

Step 1: assign each object a row vector $[a 1, a 2, \ldots, a 24]$. Generate a theoretical object of the type $2^{8} \times 3^{16}$, i.e., a matrix $A$ of $2^{8} \times 3^{16}$ row vectors.

Step 2: scan each row of matrix $A$, and mark all row vectors that satisfy the constraints.

Step 3: save all the marked row vectors as a matrix $B$ and output the matrix as the final result.

The pseudocode of the algorithm is displayed as follows.

Topological and directional relationship:

Gen (null; $R 5_{3} \mathrm{DOSa}$ )//Input: null; output: topological relationship satisfying constraints (Algorithm 1).

\section{Results and Discussion}

3.1. Comparison between $R 5_{3} D O S$-Intersection Model and MBR Model. This section proves that the R5 $5_{3} \mathrm{DOS}$-intersection model has stronger expressive power than the MBR model in the 3D space [21-23].

First, layer $R_{5}$ was defined as $R(A, B)=\mathrm{PPI}, R(A, C)=$ PPI, and $R(B, C)=\mathrm{PPI}$, and the center of outer sphere $B$ was assumed to fall into $1 \mathrm{NE}$ or $2 \mathrm{NE}$. This situation does not exist in the real world. Under Constraints 2 and 4, there is no solution to this situation. However, the $\mathrm{R} 5_{3} \mathrm{DOS}$-intersection model can explain the situation that cannot be realized in the $3 \mathrm{D}$ space.

Next, the R5 3 DOS-intersection model was found capable of expressing situation that cannot be illustrated by the MBR model through the analysis of the following example. For any three external spheres $A-C$ in the $3 \mathrm{D}$ space, it is assumed that the topological and azimuth relationships between them are known, and these spheres are separated from each other. 


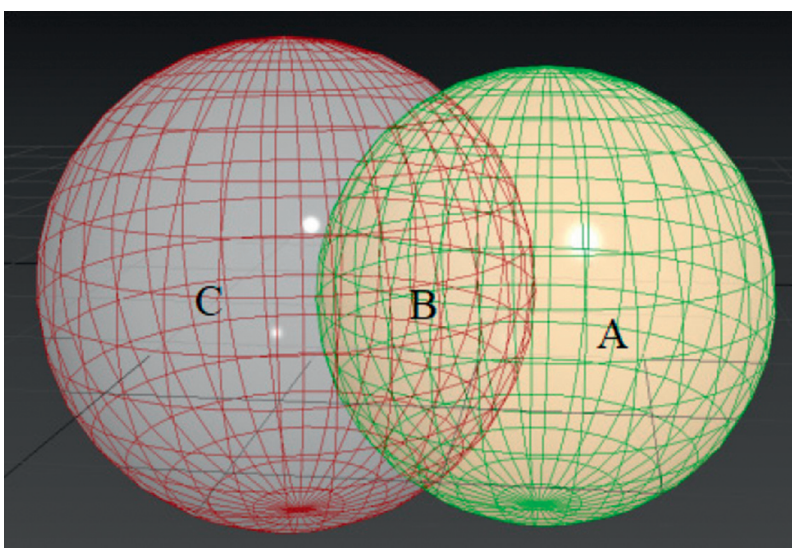

Figure 10: Special case.

For the MBR model, Example 1: (a) $\operatorname{dir}(A, B)=(1,1,1)$ and (b) $\operatorname{dir}(A, C)=(1,1,1)$ were obtained for the two examples (Figures 11 and 12).
For the R53DOS-intersection model, layer $\mathrm{R} 5$ can be described as $R(A, B)=\mathrm{DR}, R(A, C)=\mathrm{DR}$, and $R(B, C)=\mathrm{DR}$. Then,

$$
\begin{aligned}
& \left(\begin{array}{cc}
\varepsilon\left(A^{o} \cap B^{o} \cap C^{o}\right) & \varepsilon\left(A^{o} \cap\left(B^{c}\right)^{o} \cap C^{o}\right) \\
\varepsilon\left(\left(A^{c}\right)^{o} \cap B^{o} \cap C^{o}\right) & \varepsilon\left(\left(A^{c}\right)^{o} \cap\left(B^{c}\right)^{o} \cap C^{o}\right)
\end{array}\right) \vee\left(\begin{array}{cc}
\varepsilon\left(A^{o} \cap B^{o} \cap\left(C^{c}\right)^{o}\right) & \varepsilon\left(A^{o} \cap\left(B^{c}\right)^{o} \cap\left(C^{c}\right)^{o}\right) \\
\varepsilon\left(\left(A^{c}\right)^{o} \cap B^{o} \cap\left(C^{c}\right)^{o}\right) & \varepsilon\left(\left(A^{c}\right)^{o} \cap\left(B^{c}\right)^{o} \cap\left(C^{c}\right)^{o}\right)
\end{array}\right), \\
& R(A, B)=\left(\begin{array}{cc}
\varepsilon\left(A^{o} \cap B^{o}\right) & \left(\varepsilon A^{o} \cap\left(B^{c}\right)^{o}\right) \\
\varepsilon\left(\left(A^{o}\right)^{o} \cap B^{o}\right) & \varepsilon\left(\left(A^{o}\right)^{C} \cap\left(B^{c}\right)^{o}\right)
\end{array}\right)=\left(\begin{array}{llll}
0 & 1 & 1 & 1
\end{array}\right), \\
& \left(\begin{array}{cc}
\varepsilon\left(A^{o} \cap B^{o} \cap C^{o}\right) & \varepsilon\left(A^{o} \cap B^{o} \cap\left(C^{c}\right)^{o}\right) \\
\varepsilon\left(\left(A^{c}\right)^{o} \cap B^{o} \cap C^{o}\right) & \varepsilon\left(\left(A^{c}\right)^{o} \cap B^{o} \cap\left(C^{c}\right)^{o}\right)
\end{array}\right) \vee\left(\begin{array}{cc}
\varepsilon\left(A^{o} \cap\left(B^{c}\right)^{o} \cap C^{o}\right) & \varepsilon\left(A^{o} \cap\left(B^{c}\right)^{o} \cap\left(C^{c}\right)^{o}\right) \\
\varepsilon\left(\left(A^{c}\right)^{o} \cap\left(B^{c}\right)^{o} \cap C^{o}\right) & \varepsilon\left(\left(A^{C}\right)^{o} \cap\left(B^{c}\right)^{o} \cap\left(C^{c}\right)^{o}\right)
\end{array}\right), \\
& R(A, C)=\left(\begin{array}{cc}
\varepsilon\left(A^{o} \cap C^{o}\right) & \left(\varepsilon A^{o} \cap\left(C^{c}\right)^{o}\right) \\
\left(\varepsilon\left(A^{c}\right)^{o} \cap C^{o}\right) & \left(\varepsilon\left(A^{c}\right)^{C} \cap\left(C^{c}\right)^{o}\right)
\end{array}\right)=\left(\begin{array}{llll}
0 & 1 & 1 & 1
\end{array}\right), \\
& \left(\begin{array}{cc}
\varepsilon\left(A^{o} \cap B^{o} \cap C^{o}\right) & \varepsilon\left(A^{o} \cap B^{o} \cap\left(C^{c}\right)^{o}\right) \\
\varepsilon\left(A^{o} \cap\left(B^{c}\right)^{o} \cap C^{o}\right) & \varepsilon\left(A^{o} \cap\left(B^{c}\right)^{o} \cap\left(C^{c}\right)^{o}\right)
\end{array}\right) \vee\left(\begin{array}{cc}
\varepsilon\left(\left(A^{c}\right)^{o} \cap B^{o} \cap C^{o}\right) & \varepsilon\left(\left(A^{c}\right)^{o} \cap B^{o} \cap\left(C^{c}\right)^{o}\right) \\
\varepsilon\left(\left(A^{c}\right)^{o} \cap\left(B^{c}\right)^{o} \cap C^{o}\right) & \varepsilon\left(\left(A^{C}\right)^{o} \cap\left(B^{c}\right)^{o} \cap\left(C^{c}\right)^{o}\right)
\end{array}\right), \\
& R(B, C)=\left(\begin{array}{cc}
\varepsilon\left(B^{o} \cap C^{o}\right) & \varepsilon\left(B^{o} \cap\left(C^{c}\right)^{o}\right) \\
\varepsilon\left(\left(B^{c}\right)^{o} \cap C^{o}\right) & \varepsilon\left(\left(B^{o}\right)^{C} \cap\left(C^{c}\right)^{o}\right)
\end{array}\right)=\left(\begin{array}{llll}
0 & 1 & 1 & 1
\end{array}\right)
\end{aligned}
$$

Without changing the positions of $A-C$, the images of the $\mathrm{R} 5_{3} \mathrm{DOS}$-intersection model in the two examples can be obtained as Figures 13 and 14, where green, blue, and red balls are the outer spheres $\mathrm{A}-\mathrm{C}$, respectively. 


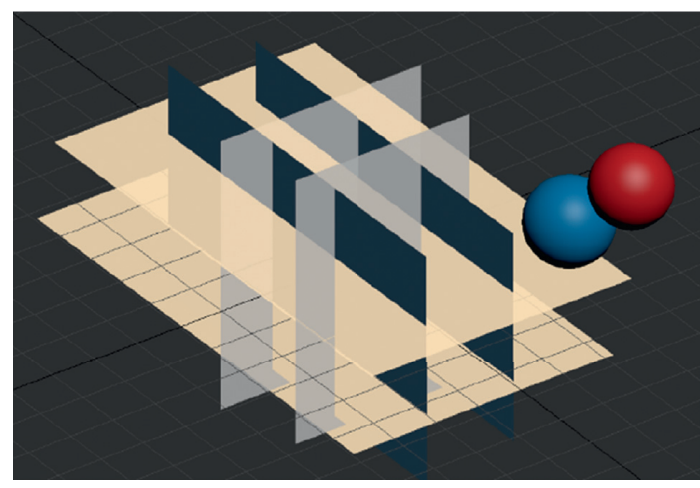

FIGURE 11: MBR model in Example 1(a).

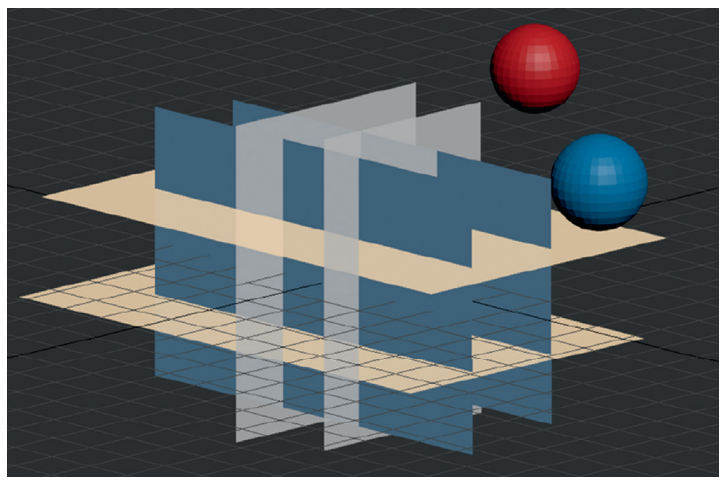

Figure 12: MBR model in Example 1(b).

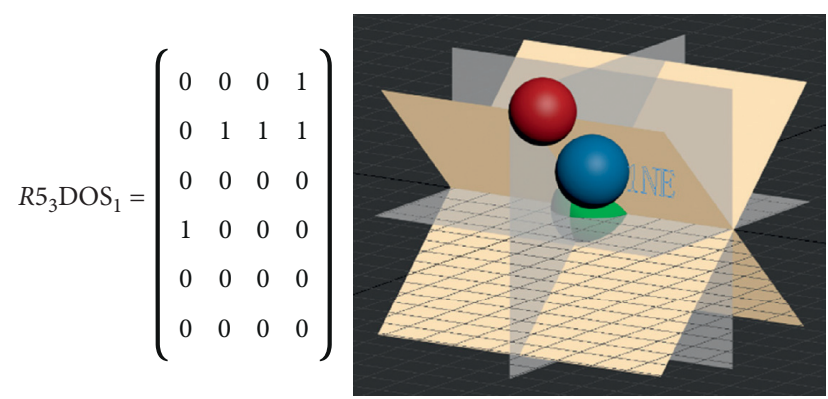

FIgURE 13: R5 ${ }_{3}$ DOS-intersection model in Example 1(a).

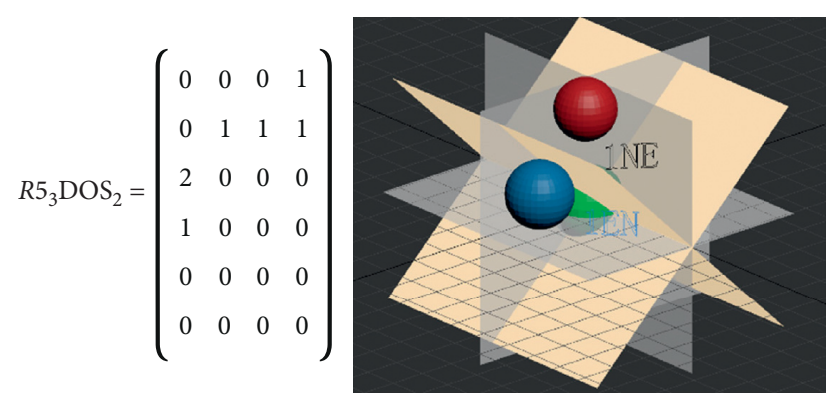

FIgURE 14: R5 ${ }_{3}$ DOS-intersection model in Example 1(b).

$$
\begin{aligned}
& R 5_{3} \mathrm{DOS}_{1}=\left(\begin{array}{llll}
0 & 0 & 0 & 1 \\
0 & 1 & 1 & 1 \\
0 & 0 & 0 & 0 \\
1 & 0 & 0 & 0 \\
0 & 0 & 0 & 0 \\
0 & 0 & 0 & 0
\end{array}\right), \\
& R 5_{3} \mathrm{DOS}_{2}=\left(\begin{array}{llll}
0 & 0 & 0 & 1 \\
0 & 1 & 1 & 1 \\
2 & 0 & 0 & 0 \\
1 & 0 & 0 & 0 \\
0 & 0 & 0 & 0 \\
0 & 0 & 0 & 0
\end{array}\right) .
\end{aligned}
$$

Example 2. (a) $\operatorname{dir}(A, B)=(0,0,1)$ and $(\mathrm{b}) \operatorname{dir}(A, C)=(0,1$, 1) were obtained for the two examples (Figures 15 and 16).

In the same way, we can get the corresponding $\mathrm{R}_{5} 3 \mathrm{DOS}$ intersection model (Figures 17 and 18):

$$
\begin{aligned}
& R 5_{3} \mathrm{DOS}_{3}=\left(\begin{array}{cccc}
1 & 0 & 0 & 0 \\
1 & 1 & 1 & 1 \\
2 & 0 & 0 & 0 \\
0 & 0 & 0 & 0 \\
0 & 0 & 0 & 0 \\
0 & 0 & 0 & 0
\end{array}\right), \\
& R 5_{3} \mathrm{DOS}_{4}=\left(\begin{array}{cccc}
1 & 0 & 0 & 0 \\
1 & 1 & 0 & 1 \\
2 & 1 & 0 & 0 \\
0 & 0 & 0 & 0 \\
0 & 0 & 0 & 0 \\
0 & 0 & 0 & 0
\end{array}\right) .
\end{aligned}
$$

Through the above comparison, it can be seen that the $\mathrm{R}_{5} 3 \mathrm{DOS}$-intersection model can represent the topological relationship of space objects $A, B$, and $C$, and it can accurately represent the spatial situation that the MBR model cannot represent.

3.2. Comnd Relationship Reasoning Based on R5 $5_{3} D O S-I n-$ tersection Model. This section applies the R5 $5_{3}$ DOS-Intersection Model to the reasoning of the compound relationships between simple regions in the $3 \mathrm{D}$ space. It is assumed that the topological and azimuth relationships between simple regions $A$ and $B$ and those between simple regions $B$ and $C$ are known in advance. Then, the goal is to deduce the possible topological and azimuth relationships between simple regions $A$ and $C$.

According to Section 2.3, we have 


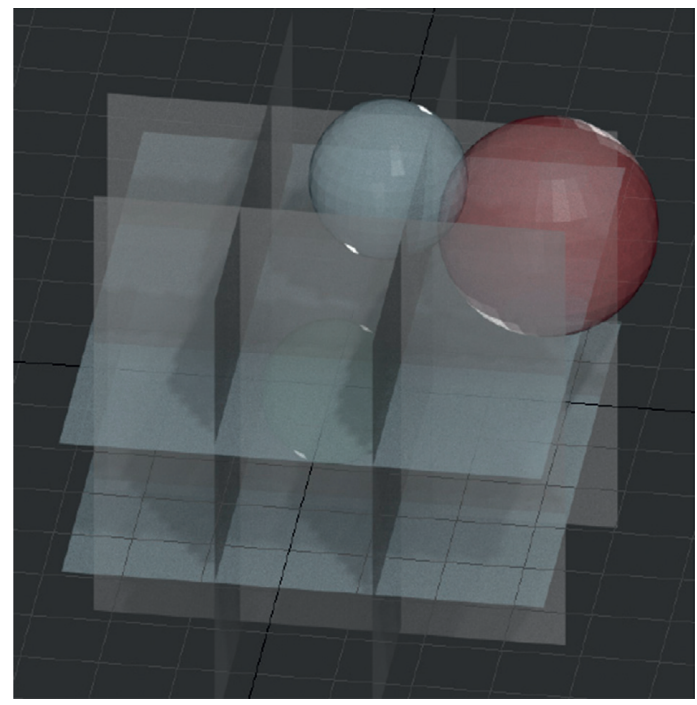

Figure 15: MBR model in Example 2(a).

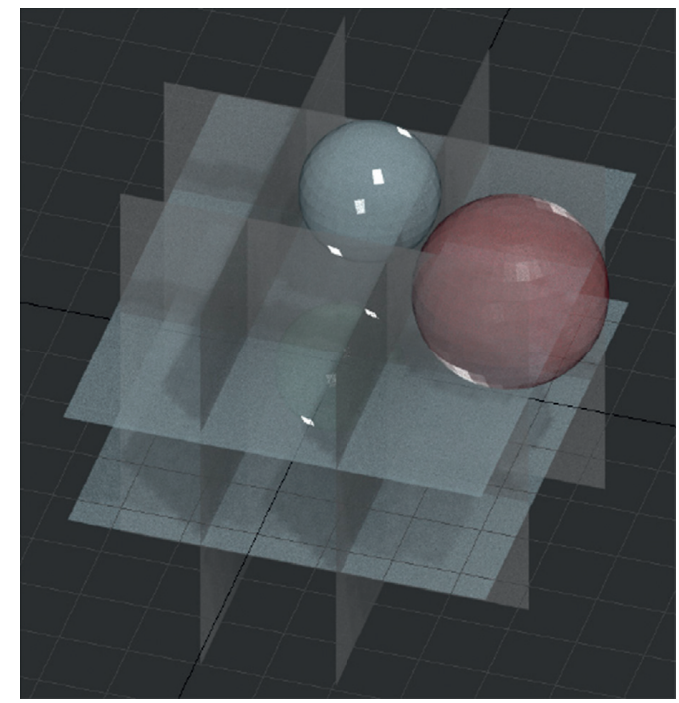

Figure 16: MBR model in Example 2(b).

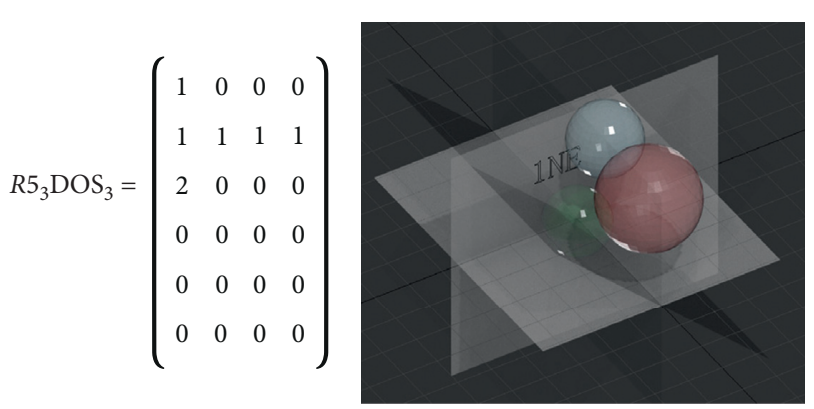

FIgURE 17: R5 ${ }_{3}$ DOS-intersection model in Example 2(a).

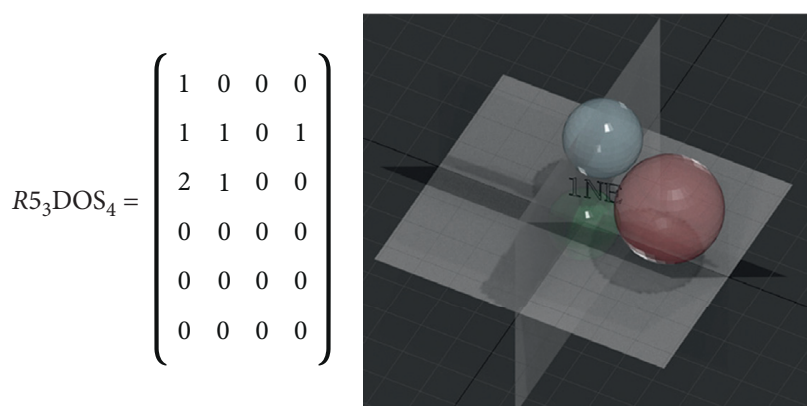

FIGURE 18: R5 ${ }_{3}$ DOS-intersection model in Example 2(a). 
TABLE 3: The list of all directional and topological relationships.

\begin{tabular}{|c|c|c|}
\hline Topological relationships & & Regions \\
\hline DR & & $\begin{array}{l}\text { DRs1NE, DRs2NE, DRs1EN, DRs2EN, DRs5ES, DRs6ES, DRs5SE, } \\
\text { DRs6SE, DRs8SW, DRs7SW, DRs8WS, DRs7WS, } \\
\text { DRs4WN, DRs3WN, DRs4NW, DRs3NW } \\
\text { POs1NE, POs2NE, POs1EN, POs2EN, POs5ES, POs6ES, POs5SE, POs6SE, } \\
\text { POs8SW, POs7SW, POs8WS, POs7WS, } \\
\text { POs4WN, POs3WN, POs4NW, POs3NW }\end{array}$ \\
\hline $\begin{array}{l}\text { PP } \\
\text { EQ }\end{array}$ & & $\begin{array}{l}\text { PPs1NE, PPs2NE, PPs1EN, PPs2EN, PPs5ES, PPs6ES, PPs5SE, } \\
\text { PPs6SE, PPs8SW, PPs7SW, PPs8WS, } \\
\text { PPs7WS, PPs4WN, PPs3WN, PPs4NW, PPs3NW } \\
\text { Equal }\end{array}$ \\
\hline PPI & & $\begin{array}{l}\text { PPIs1NE, PPIs2NE, PPIs1EN, PPIs2EN, PPIs5ES, PPIs6ES, } \\
\text { PPIs5SE, PPIs6SE, PPIs8SW, PPIs7SW, PPIs8WS, } \\
\text { PPIs7WS, PPIs4WN, PPIs3WN, PPIs4NW, PPIs3NW }\end{array}$ \\
\hline$R(B, C)$ & $R(A, B)$ & $R(A, C)$ \\
\hline $\mathrm{DR}(B, C)$ & $\begin{array}{l}\text { all }(A, B) \\
\text { POall }(A, B) \\
\text { PPall }(A, B) \\
\text { EQall }(A, B) \\
\text { PPIall }(A, B)\end{array}$ & 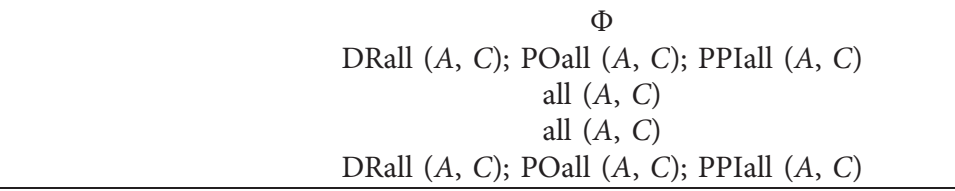 \\
\hline $\mathrm{PO}(B, C)$ & $\begin{array}{l}\text { DRall }(A, B) \\
\text { POall }(A, B) \\
\text { PPall }(A, B) \\
\text { EQall }(A, B) \\
\text { PPIall }(A, B)\end{array}$ & 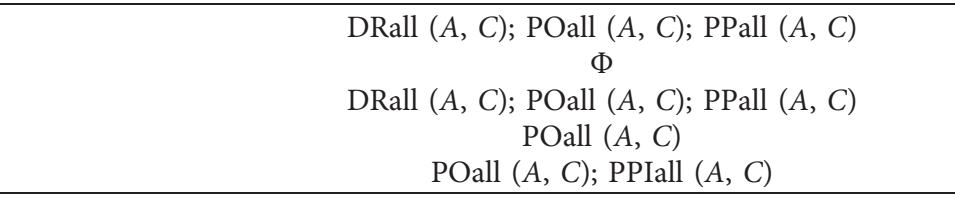 \\
\hline $\mathrm{PP}(B, C)$ & $\begin{array}{l}\text { DRall }(A, B) \\
\text { POall }(A, B) \\
\text { PPall }(A, B) \\
\text { EQall }(A, B) \\
\text { PPIall }(A, B)\end{array}$ & $\begin{array}{c}\text { DRall }(A, C) ; \text { POall }(A, C) ; \text { PPall }(A, C) \\
\text { POall }(A, C) ; \text { PPall }(A, C) \\
\text { PPall }(A, C) \\
\text { PPall }(A, C) \\
\text { POall }(A, C) ; \text { EQall }(A, C) ; \text { PPall }(A, C) ; \text { PPIall }(A, C)\end{array}$ \\
\hline EQ $(B, C)$ & $\begin{array}{l}\text { DRall }(A, B) \\
\text { POall }(A, B) \\
\text { PPall }(A, B) \\
\text { EQall }(A, B) \\
\text { PPIall }(A, B)\end{array}$ & $\begin{array}{c}\text { all }(A, C) \\
\text { POall }(A, C) \\
\text { PPall }(A, C) \\
\text { EQall }(A, C) \\
\text { PPIall }(A, C)\end{array}$ \\
\hline PPI $(B, C)$ & $\begin{array}{l}\text { DRall }(A, B) \\
\text { POall }(A, B) \\
\text { PPall }(A, B) \\
\text { EQall }(A, B) \\
\text { PPIall }(A, B)\end{array}$ & $\begin{array}{c}\text { DRall }(A, C) \\
\text { DRall }(A, C) ; \text { POall }(A, C) \text {; PPIall }(A, C) \\
\Phi \\
\text { PPIall }(A, C) \\
\text { PPIall }(A, C)\end{array}$ \\
\hline
\end{tabular}

$$
R 5_{2} \mathrm{DOS}=\left(\begin{array}{cccc}
A^{o} \cap B^{o} & A^{o} \cap\left(B^{c}\right)^{o} & \left(A^{c}\right)^{o} \cap B^{o} & \left(A^{c}\right)^{o} \cap\left(B^{c}\right)^{o} \\
s 1 \mathrm{NE} & s 2 \mathrm{EN} & s 3 \mathrm{NW} & s 4 \mathrm{NW} \\
s 1 \mathrm{EN} & s 2 \mathrm{EN} & s 3 \mathrm{NW} & s 4 \mathrm{NW} \\
s 5 \mathrm{ES} & s 6 \mathrm{ES} & s 7 \mathrm{WS} & s 8 \mathrm{WS} \\
s 5 \mathrm{SE} & s 6 \mathrm{SE} & s 7 \mathrm{SW} & s 8 \mathrm{SW}
\end{array}\right) .
$$

Using the $\mathrm{R}_{3} \mathrm{DOS}$-intersection model, a total of 65 topological and azimuth relationships were obtained from the real world. Hence, it is possible to obtain 65 0-1 matrices of 5 rows and 4 columns, which is denoted as $\Omega_{1}=\left\{R_{i} ; i=1, \ldots, 65\right\} R_{i}$. Targeting at region $\mathrm{A}$, the topological and directional relationships between $A$ and $C$ and those between $B$ and $C$ were taken into account.

Since the topological and azimuth relationships between simple regions $A$ and $B$ and those between simple regions $B$ and $C$ are known in advance, we have $R(B, C) \in \Omega_{1}$. Then, the possible topological and orientation relationships between $A$ and $C$ were derived from the R5 ${ }_{3}$ DOS-intersection model. According to Definition 2, we have 


$$
\begin{aligned}
&\left(\begin{array}{cc}
\varepsilon\left(A^{o} \cap B^{o} \cap C^{o}\right) & \varepsilon\left(A^{o} \cap\left(B^{c}\right)^{o} \cap C^{o}\right) \\
\varepsilon\left(\left(A^{c}\right)^{o} \cap B^{o} \cap C^{o}\right) & \varepsilon\left(\left(A^{c}\right)^{o} \cap\left(B^{c}\right)^{o} \cap C^{o}\right)
\end{array}\right) \vee\left(\begin{array}{cc}
\varepsilon\left(A^{o} \cap B^{o} \cap\left(C^{c}\right)^{o}\right) & \varepsilon\left(A^{o} \cap\left(B^{c}\right)^{o} \cap\left(C^{c}\right)^{o}\right) \\
\varepsilon\left(\left(A^{c}\right)^{o} \cap B^{o} \cap\left(C^{c}\right)^{o}\right) & \varepsilon\left(\left(A^{C}\right)^{o} \cap\left(B^{c}\right)^{o} \cap\left(C^{c}\right)^{o}\right)
\end{array}\right), \\
& R(A, B)=\left(\begin{array}{cc}
\varepsilon\left(A^{o} \cap B^{o}\right) & \varepsilon\left(A^{o} \cap\left(B^{c}\right)^{o}\right) \\
\varepsilon\left(\left(A^{c}\right)^{o} \cap B^{o}\right) & \varepsilon\left(\left(A^{o}\right)^{C} \cap\left(B^{c}\right)^{o}\right)
\end{array}\right), \\
&\left(\begin{array}{cc}
\varepsilon\left(A^{o} \cap B^{o} \cap C^{o}\right) & \varepsilon\left(A^{o} \cap B^{o} \cap\left(C^{c}\right)^{o}\right) \\
\varepsilon\left(A^{o} \cap\left(B^{c}\right)^{o} \cap C^{o}\right) & \varepsilon\left(A^{o} \cap\left(B^{c}\right)^{o} \cap\left(C^{c}\right)^{o}\right)
\end{array}\right) \vee\left(\begin{array}{cc}
\varepsilon\left(\left(A^{c}\right)^{o} \cap B^{o} \cap C^{o}\right) & \varepsilon\left(\left(A^{c}\right)^{o} \cap B^{o} \cap\left(C^{c}\right)^{o}\right) \\
\varepsilon\left(\left(A^{c}\right)^{o} \cap\left(B^{c}\right)^{o} \cap C^{o}\right) & \varepsilon\left(\left(A^{C}\right)^{o} \cap\left(B^{c}\right)^{o} \cap\left(C^{c}\right)^{o}\right)
\end{array}\right), \\
& R(B, C)=\left(\begin{array}{cc}
\varepsilon\left(B^{o} \cap C^{o}\right) & \varepsilon\left(B^{o} \cap\left(C^{c}\right)^{o}\right) \\
\varepsilon\left(\left(B^{c}\right)^{o} \cap C^{o}\right) & \left.\varepsilon\left(B^{o}\right)^{C} \cap\left(C^{c}\right)^{o}\right)
\end{array}\right) .
\end{aligned}
$$

Suppose the real-world 0-1 matrices satisfy

$$
M=\left\{M_{i}=1, \ldots, n\right\},
$$

Then, all 0-1 matrices must meet:

$$
m_{i}=R(A, B) \vee R(B, C), \quad i=1, \ldots, n .
$$

Hence, the matrix that does not satisfy the condition belongs to the empty set, namely, $M \in \varnothing$. This shows the topological and directional relationships $R(A, B)$ and $R(A$, $C)$ cannot be compounded. Then, all 0-1 matrices represented in the R $5_{3} \mathrm{DOS}$-intersection model were judged one by one. The duplicates in the set $\left\{M_{i}=1, \ldots, n\right\}$ were removed, leaving the possible topological and azimuth relationships between $A$ and $C$.

In theory, there are a total of $65 \times 65=4,225$ topologicalazimuth relationships $R(A, B)$ and $R(B, C)$. On this basis, the compound relationship reasoning table was set up (Table 4).

\section{Conclusions}

This paper extends the compound directional and topological relationships on the $2 \mathrm{D}$ plane to the $3 \mathrm{D}$ space and then creates the R53DOS-intersection model. Based on the model, a total of 11,038 directional and topological relationships were calculated. Compared with the MBR model, the proposed model can describe the relationships between simple regions accurately and express the relationships with sufficient clarity. To further improve the model, the future research will consider the impact of simple area boundaries on the model and apply the R5DOS model to the formation control of UAV formations.

\section{Data Availability}

The data used to support the findings of the study are available from the corresponding author upon request.

\section{Conflicts of Interest}

The authors declare that they have no conflicts of interest.

\section{Acknowledgments}

This work was supported by National Natural Science Foundation of China (Grant nos. 41601454 and 41671397), Science and Technology Development Project of Jilin Province China (Grant no. 20191001008XH), Science Foundation of Jilin Provincial Education Department China (Grant nos. JJKH20200329KJ and JJKH20190922KJ), Development and Reform Commission Project of Jilin Province China (Grant no. 2020C037-7), and Ecology and Environment Department Project of Jilin Province China (Grant no. 2019-02).

\section{Supplementary Materials}

This code is the screening algorithm of the R5DOS-intersection model. The purpose is to screen several matrices theoretically in the model according to the constraints and finally get the algorithm of the matrix that meets the requirements, the result of running the code needs simple processing, not the result of the article. The code is developed based on MATLAB software. (Supplementary Materials)

\section{References}

[1] L. Wang, L. Zhao, G. Huo et al., "Visual semantic navigation based on deep learning for indoor mobile robots," Complexity, vol. 2018, Article ID 1627185, 12 pages, 2018.

[2] Y. Fan, K. Xing, X. Jiang et al., 'Fuzzy adaptation algorithms' control for robot manipulators with uncertainty modelling errors," Complexity, vol. 2018, Article ID 5468090, 8 pages, 2018.

[3] J. Li, K. Deng, X. Huang, and J. Xu, "Analysis and applications of location-aware big complex network data," Complexity, vol. 2019, Article ID 3410262, 2 pages, 2019.

[4] D. A. Randell and T. Cohn, Modelling Topological and Metrical Properties, University of Warwick, Coventry, UK, 1989.

[5] D. A. Randell, Z. Cui, and A. G. Cohn, "A spatial logic based on regions and connection," in Proceedings of the $3 \mathrm{rd} \mathrm{In-}$ ternational Conference on Principles of Knowledge Representation, pp. 165-176, Cambridge, MA, USA, 1992.

[6] M. J. Egenhofer and R. D. Franzosa, "On the equivalence of topological relations," International Journal of Geographical Information Systems, vol. 9, no. 2, pp. 133-152, 1995. 
[7] M. J. Egenhofer and R. D. Franzosa, "Point-set topological spatial relations," International Journal of Geographical Information Systems, vol. 5, no. 2, pp. 161-174, 1991.

[8] S. Li, Research on Orientation Relations and Integrative Reasoning with Topological Relations and Orientation Relations in Dynamic Settings, Jilin University, Changchun, China, 2013.

[9] Z. Chen, X. Liu, J. Yang, E. Little, and Y. Zhou, "Deep learning-based method for sem image segmentation in mineral characterization, an example from duvernay shale samples in western Canada Sedimentary Basin," Computers \& Geosciences, vol. 138, Article ID 104450, 2020.

[10] X. Li, C. Lin, and X. Xu, "A target tracking model for enterprise production monitoring system based on spatial information and appearance model," Traitement du Signal, vol. 36, no. 4, pp. 369-375, 2019.

[11] F. Lei, J. Cai, Q. Dai et al., "Deep learning based proactive caching for effective wsn-enabled vision applications," Complexity, vol. 2019, Article ID 5498606, 12 pages, 2019.

[12] B. Beirami and M. Mokhtarzade, "Spatial-spectral random patches network for classification of hyperspectral images," Traitement du Signal, vol. 36, no. 5, pp. 399-406, 2019.

[13] M. Z. Huang, D. Tian, and H. B. Liu, "A hybrid fuzzy wavelet neural network model with self-adapted fuzzy -means clustering and genetic algorithm for water quality prediction in rivers," Complexity, vol. 2018, Article ID 8241342, 11 pages, 2018.

[14] Z. Li, C. Xu, and C. Liu, "Frequent subtree mining algorithm for ribonucleic acid topological pattern," Revue d'Intelligence Artificielle, vol. 33, no. 1, pp. 75-80, 2019.

[15] Q. Liu, X. He, F. Guan et al., "Method and implementation of improving the pointing accuracy of an optical remote sensor using a star sensor," Traitement du Signal, vol. 36, no. 4, pp. 311-317, 2019.

[16] Z. H. Wang, H. W. Yan, and Y. C. Yang, "Compound spatial query based on direction and distance relation," Engineering of Surveying \& Mapping, vol. 23, no. 11, pp. 7-12, 2014.

[17] Y. Liu, X. Gong, and D. Kong, "Spatial reasoning based on 3DICSRM model," Mathematical Problems in Engineering, vol. 2019, Article ID 2892545, 9 pages, 2019.

[18] R. Hou, T. Wu, and J. J. Yang, "Reasoning with cardinal directions in 3D space based on block algebra," in Proceedings of the International Conference on Electronic Information Technology and Intellectualization, pp. 500-511, Guangzhou, China, June 2016.

[19] S. Wang, R. Dong, W. Song, and C. Wang, "Qualitative spatial reasoning with oriented point relation in 3D space," Chinese Journal of Electronics, vol. 28, no. 2, pp. 325-330, 2019.

[20] M. A. Cobb, F. E. Petry, and K. B. Shaw, "Fuzzy spatial relationship refinements based on minimum bounding rectangle variations," Fuzzy Sets and Systems, vol. 113, no. 1, pp. 111-120, 2000.

[21] Y. B. He and J. Bian, "Research on the model of spatial direction relation in spatial reasoning," Computer \& Digital Engineering, vol. 38, no. 4, pp. 62-65, 2010.

[22] S.-S. Yu, S.-W. Chu, C.-M. Wang, Y.-K. Chan, and T.-C. Chang, "Two improved k-means algorithms," Applied Soft Computing, vol. 68, pp. 747-755, 2018.

[23] C. L. Sabharwal and J. L. Leopold, "Cardinal direction relations in qualitative spatial reasoning," International Journal of Computer Science and Information Technology, vol. 6, no. 1, pp. 1-13, 2014. 